\title{
Correction to: Investigating changes in $\beta$-adrenergic gene expression (ADRB1 and ADRB2) in Takotsubo (stress) cardiomyopathy syndrome; a pilot study
}

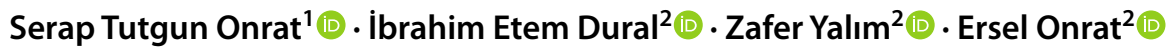

Published online: 20 February 2022

(c) Springer Nature B.V. 2022

\section{Correction to: \\ Molecular Biology Reports (2021) 48:7893-7900 \\ https://doi.org/10.1007/s11033-021-06816-w}

\section{Dear Editor,}

To clarify our methods we respectfully submit our statements regarding our research so that our work can be better understood.

We have done long-read readings in the gene region we are targeting in order to illuminate the possible genetic causes of Takotsubo Syndrome by using a different and new method. It is very valuable to conduct research on a disease that we call orphan diseases and unfortunately, because the commercial results are not high, pharmaceutical companies and the health product development industry are not interested. Our study is a well-designed and finalized study, and it is economical for its budget.

The original article can be found online at https://doi.org/10.1007/ s11033-021-06816-w.

Serap Tutgun Onrat

tutgunonrat@yahoo.com; serap.onrat@afsu.edu.tr

İbrahim Etem Dural

iedural@hotmail.com; etem.dural@afsu.edu.tr

Zafer Yalım

zaferyalm@yahoo.com.tr; zafer.yalim@afsu.edu.tr

Ersel Onrat

eonrat@yahoo.com; ersel.onrat@afsu.edu.tr

1 Department of Medical Genetics, Afyonkarahisar University of Health Sciences Faculty of Medicine, Zafer Sağllk Külliyesi B Blok, Kat:1, Dörtyol Mah. 2078 Sok. No:3, 03200 Afyonkarahisar, Turkey

2 Department of Cardiology, Afyonkarahisar University of Health Sciences Faculty of Medicine, Afyonkarahisar, Turkey
Targeted sequencing by enrichment of target DNA/RNA molecules or depletion of unwanted molecules is a valuable method of generating sufficient depth of coverage for regions of interest, for informative and cost-effective analysis. Targeted, long nanopore sequencing reads enable entire genes and genomic regions of interest to be fully characterized, allowing researchers to look beyond a handful of known variants. This can include novel variants in gene promoters, introns, and repetitive regions, plus the analysis of structural variants, base modifications, and phasing.

"Gene expression" in the sentence in Abstract Section may have caused misunderstanding and it would be more descriptive to use "gene variation";

"The gene variations of cardiac $\beta-1$ ADRB1 (rs 1801253ENST00000369295.4), G > C, (Gly389Arg) and cardiac $\beta$-2 ADRB2 (rs1800888-ENSG00000169252), C > T (Thr1651le) adrenoceptors were investigated".

Since our published article was presented as a pilot study, we published the results of variant analysis using DNA in the first stage in our article. The result we give in this article is the variant analysis result obtained by the "long read" reading of our study. Expression analysis with RNA extraction is the next step our project, when project completely finished we will plan to prepare new article.

The study was supported by grants from the Afyonkarahisar Health Science University Research Project Commission and the project number is 20.GENEL.006. We did not change the project title and abstract. We wanted this to be a catchy topic because genetic variations can alter gene expression and we give result for variants part of our research in this article which was pilot study. Genetic variations in gene regions can cause disruption of genomic balance. Thus, gene expression changes may occur with the disruption of this balance. We used the definition of "gene expression" as a word, because we didn't change the project title in our article, not to describe the method we used. The part of our project described in this article is variant 
analysis, we used DNA, not RNA, in our study since there was no expression study. According to the method used, the study was completed according to the DNA-based protocol, and variants were searched by bioinformatic analysis, and no variants were found.

When the final report of the completed study is examined, it is seen that it is a sequencing study that aims to detect variants by sequencing certain regions of ADRB1 and ADRB2 genes using Oxford Nanopore Technology. It is possible to define the study methodologically as a genotyping study for the targeted regions. The sequence analysis method is generally used for such variant discovery studies. There are three main sequencing technologies currently in active use: 1st Generation (Sanger sequencing), 2nd Generation (Next generation sequencing), and 3rd Generation (Pacbio, Oxford Nanopore Sequencing).

First-generation sequencing technology, also known as Sanger sequencing technology, is the oldest and still gold standard automated sequencing technology. However, in addition to the high cost of sequence per base, they also pose problems such as a high repetition rate. Next-generation sequence analysis, also called second-generation sequence analysis, is a technology that can perform hundreds of thousands or even millions of readings at the same time. With the new generation sequencing analysis, molecular analysis of many genes can be performed in more than one patient at the same time. Today, next-generation sequencing analysis has been widely used in research laboratories and clinical diagnosis centers. With next-generation sequence analysis, whole genome, whole exome, or targeted gene analyzes can be performed. Despite all these advantages, the next-generation sequencing method has some shortcomings. Sequence errors and misalignment can result, especially when the read depth is low. In addition, although the cost of reading and analysis per base with next-generation sequence analysis is lower than Sanger sequence analysis, the initial setup of the device required for analysis is quite costly. To reduce the cost per sample, simultaneous analysis of multiple cases is recommended after accumulating DNA samples of cases (pool optimization). This both causes a waste of time and is not a cost-effective solution for centers with less than a certain number of tests.

The rapid advances in next-generation sequence analysis technology and the recognition of existing shortcomings continue with the introduction of new technologies into the field of application. The "Oxford Nanopore" technology, which is defined as the third generation sequence analysis -even the fourth generation in some publications- provides all the benefits such as the analysis of many regions at the same time, low cost per base, brought by the new generation sequence analysis technology, long reads, fast analysis and especially thanks to its small dimensions, it allows use in the field, outside the laboratory and at the beginning of the case.
Using nanopore sequencing technology, a single DNA or Ribo Nucleic Acid molecule can be sequenced without the need for amplification or chemical labeling of the sample. This technology has so far been used in many areas such as rapid identification of viral pathogens, especially Covid19, monitoring of Ebola, monitoring of food safety, human genome sequencing, plant genome sequencing, antibiotic resistance monitoring and haplotyping.

The Oxford Nanopore Technology used in the study was a reasonable choice as it is the most up-to-date sequence analysis technology and can achieve very high read numbers in a short time. In addition, there is no study in the literature that sequences ADRB1 and ADRB2 genes with this technology. This adds methodological originality to the study. If Next Generation Sequence Analysis was used instead of Oxford Nanopore Sequencing in this study, similarly higher reads and even lower error rates could be obtained. However, the single run cost of even the lowest-capacity Next Generation Sequence Analysis instrument (Illumina iSeq, MiniSeq) exceeded the overall budget of the study, along with consumables and cartridge prices.

As a result of the study, it is seen that no variant was detected in the determined exonic regions of the ADRB1 gene. This possibility is inherent in all such variant discovery studies. In addition, raw data from the Oxford Nanopore Sequencing process are available and can be reanalyzed if desired. In case of any doubt, the data will be examined by experts in this field and transparency will be easily ensured.

\section{Materials and methods}

\section{Patients}

Within the framework of the project; 10 patients who were admitted to Afyonkarahisar Health Sciences University, Faculty of Medicine, Department of Cardiology and hospitalized with the diagnosis of acute coronary syndrome or acute heart failure and diagnosed with Takotsubo Cardiomyopathy according to the diagnostic criteria of Mayo Clinic TK, included in the study. As the control group, 10 healthy individuals included in the study. Medical history, physical examination, laboratory findings, electrocardiography, echocardiography, coronary angiography and ventriculography of the patients were evaluated. $10 \mathrm{ml}$ of venous blood samples taken from the forearm with the vacutainer system from the patients were transferred to the Medical Genetics Department on the same day. This study was performed according to the Declaration of Helsinki II. All participants provided and signed informed consent and were approved by the Institutional Local Ethics Committee.

One of the 20 subjects (10 patients and 10 controls) diagnosed with Takotsubo Cardiomyopathy included in the 
study did not want to donate blood. Genomic DNA (gDNA) was obtained from a total of 18 cases ( 9 patients and 9 controls) using the QIAamp DNA Blood Mini Kit (Qiagen) and quantified with the NanoDrop ND-1000 Spectrophotometer. Therefore, the number of control groups was started to be 9 to equalize the number of study and control groups. In our study group, menopausal women are more affected by Takotsubu cardiomyopathy, so the number of male cases is less. The characteristics of the demographic and clinical variables of the study and control groups are given in Table 1, and the echocardiographic results of our study and control groups are given in Table 2.

\section{Library prep with native barcoding amplicons}

Library preparation was completed using the Native Barcoding Kit 1D (catalog number EXP-NBD104) and Ligation Sequencing Kit (catalog number SQK-LSK109; Oxford Nanopore Technologies, Oxford, UK) (Fig. 1b). ONT libraries were prepared as follows: $48 \mu$ l of genomic DNA per library was $3.5 \mu \mathrm{l}$ of NEBNext FFPE DNA Repair Buffer, $2 \mu \mathrm{l}$ of NEBNext FFPE DNA Repair Mix (New England Biolab, M6630), and $3.5 \mu \mathrm{l}$ of nuclease-free water (NFW) and incubated at $20^{\circ} \mathrm{C}$ for 15 min for DNA repair, re-pooled and cleaned up using a $0.8 \times$ volume of AMPure XP beads (Beckman Coulter) according to the manufacturer's instructions, with final elution in $30 \mu \mathrm{l}$ of $\mathrm{EB}(10 \mathrm{mM}$ Tris $\mathrm{pH}$ 8.0).

The DNA recovered from the elution well $(\sim 40 \mu \mathrm{l})$ was brought to $50 \mu \mathrm{l}$ and end-repaired by the addition of $3.5 \mu \mathrm{l}$ NEBNext Ultra II End Prep Reaction Buffer and $3 \mu 1$ NEBNext Ultra II End Prep Enzyme Mix (New England Biolab, E7546) with incubation for $5 \mathrm{~min}$ at $20^{\circ} \mathrm{C}$ followed by 5 min at $65^{\circ} \mathrm{C}$. The sample was cleaned up with $1 \times$ volume AMPure XP beads and eluted in $30 \mu \mathrm{l}$ of EB.

PCR adapters $(20 \mu \mathrm{l})$ from Oxford Nanopore Technology (SQK-LSK109 Ligation Sequencing Kit 1D) were ligated to the end-repaired DNA with $50 \mu \mathrm{l}$ of NEB Blunt/TA Ligase Master Mix (M0367) at room temperature for $10 \mathrm{~min}$, followed by clean-up with $1 \times$ volume AMPure XP beads and elution in $48 \mu \mathrm{l}$ of EB.

Combined $22.5 \mu \mathrm{L}$ of purified DNA, $2.5 \mu \mathrm{L}$ of Native Barcode and $25 \mu \mathrm{L}$ of blunt/TA ligase master mixe in a new $1.5 \mathrm{~mL}$ DNA-low binding reaction tube, mix was by tapped and spined briefly. It was incubated for $10 \mathrm{~min}$ at room temperature. Then $65 \mu \mathrm{L}$ pooled barcoded DNA, $5 \mu \mathrm{L}$ Adapter MixII (AMII), $20 \mu \mathrm{L}$ NEBNext Quick Ligation Buffer (5x) and $10 \mu \mathrm{L}$ Quick T4 DNA Ligase were prepared for Adaptor ligation and clean-up steps, and mix was gently by flicked the tube and spin down. Finally, $61.5 \mathrm{ng} / \mathrm{ml}$ of the control group and $58.8 \mathrm{ng} / \mathrm{ml}$ of the patient group's pooled DNA were washed twice with $250 \mu \mathrm{L}$ of Short Fragment Buffer (SFB) Buffer and loaded into the MinION device by adding $15 \mu \mathrm{L}$ of Elution Buffer. In a new tube, prepared the library for loading as follows; $37.5 \mathrm{ml}$ Sequencing Buffer,(SQB), $25.5 \mathrm{ml}$ Loading Beads, 14 ml DNA library. Then gently loaded SpotOn Flowcell. As the first operation; Flow-Check was performed to check pore numbers, then samples were loaded separately as study and control groups using the MinION Mk1b sequencer with two FLO-MINSP6 flow cells. Assuming the sample count as 10 , respectively, $1 \mathrm{M}$ reads with an average length of 100,000 bases were obtained. Raw data (FAST5 files) were obtained using MinKNOW software version 21.06.0 (Oxford Nanopore Technologies), which were converted to FASTQ files and sorted into each sample depending on their barcode sequences by using Guppy software version 4.4.0 (Oxford Nanopore Technologies).

\section{Data analysis}

The data obtained as a result of MinION (Oxford Nanopore Technologies) sequencing were processed in fast 5 format. In the first stage, basecalling and demultiplexing operations of fast5 files were performed with Guppy basecaller 4.4.0 software, and the obtained sequences were obtained in fastq format and separated into barcodes. Sequences in Fastq format were loaded into the Geneious Prime 2021.0.3 (www.geneious.com) program in separate files according to each barcode, and the sequences consisting of different fragments with the same barcode were combined. The sequences were made ready for the mapping process by performing filtering processes according to the quality scores of the obtained sequences. The reference sequence required for the mapping process was obtained after the analysis of the presented primers. Given for the ADRB1 gene; forward 5'-AGACGTGCTATGTGTGACGG-3' and reverse 5'-AGC ACTTGGGGGTCGTTGTAG-3' primer pair, given for the ADRB2 gene; forward 5'-TGGATTGTGTCGGGCCTTA-3' and reverse 5'-TGGCACGGTACCAGTGCAT-3' PCR was performed.

\section{Limitations}

The major limitation of this study was the small sample size and single-center design. Although we collaborated with many different clinics to identify these patients, our sample size was small, possibly due to the Covid-19 pandemic. Nevertheless, we think that our study is important because our data show the frequency of this disease in our city and provide pilot data to investigate the effect of $\beta$-Adrenergic gene variation alteration.

Publisher's Note Springer Nature remains neutral with regard to jurisdictional claims in published maps and institutional affiliations. 